\title{
The Relationship Between Grit and Resilience in Emergency Medical Service Personnel
}

\author{
Mandi Musso, PhD, Danielle Tatum, PhD, Diana Hamer, PhD, Rebecca Hammarlund, PhD, Leslie Son, PhD, \\ Pamela McMahon, PhD, MPH
}

Division of Academic Affairs, Our Lady of the Lake Regional Medical Center, Baton Rouge, LA

\begin{abstract}
Background: Emergency medical service (EMS) personnel are regularly exposed to traumatic incidents and experience higher rates of symptoms of posttraumatic stress disorder (PTSD) than the general population. Grit is a construct proposed to be associated with achievement, but it has demonstrated preliminary evidence of an association with resilience. The current study examined the relationship between grit and resilience among EMS workers.

Methods: A link to an online survey was sent to East Baton Rouge Parish Emergency Medical Services personnel via an email distribution list. Demographic variables and the following self-report measures were assessed: the PTSD Checklist for DSM-5 (PCL-5), the Grit Scale, the Brief COPE scale, and the Professional Exposure to Traumatic Experiences scale (modified from the Life Events Checklist).

Results: PCL-5 scores were significantly and negatively correlated with the Grit Scale score $(r=-0.57, P<0.01)$. Hierarchical regression revealed that grit and coping mechanisms were predictive of self-reported PTSD symptoms (adjusted $R^{2}=68.7 \%$, $F(15,67)=9.81, P<0.001)$. Examination of the coefficients revealed that lower total Grit Scale scores and higher scores on the following Brief COPE scales were significant predictors of PCL-5 scores: denial, substance abuse, disengagement, and self-blame.

Conclusion: This study examined the relationship between grit and resilience, measured by self-reported PTSD symptoms. Our results demonstrate a significant relationship between grit and resilience. Grit is related to, but distinct from, other constructs that predict resilience, such as coping mechanisms. Large prospective studies could have significant implications for hiring practices and building grit in existing personnel to bolster resilience.
\end{abstract}

Keywords: Emergency medical services, emergency medical technicians, emergency responders, stress disorders-posttraumatic, resilience-psychological

Address correspondence to Mandi Musso, PhD, Division of Academic Affairs, Our Lady of the Lake Regional Medical Center, 5246 Brittany Dr., Baton Rouge, LA 70808. Tel: (225) 757-4148. Email: mmuss1@/suhsc.edu

\section{INTRODUCTION}

Emergency medical technicians (EMTs) and paramedics are regularly exposed to traumatic incidents, including violence, suicide, severe injuries, and death. In one survey, $82 \%$ of emergency medical service (EMS) personnel surveyed reported experiencing a particularly disturbing event. ${ }^{1}$ Studies have estimated that $9 \%$ to $22 \%$ of EMTs/paramedics develop symptoms of posttraumatic stress disorder (PTSD), ${ }^{2}$ and a 2018 metanalysis of 30,878 ambulance personnel estimated the prevalence rate of PTSD to be $11 \% .^{3}$ These estimates are higher than the rates of PTSD found in the general population. ${ }^{2-4}$ PTSD is a clinical disorder that can occur after an individual is exposed to traumatic events, either directly or indirectly. ${ }^{5}$ Symptoms of PTSD include reexperiencing the event, avoidance of trauma-related stimuli, negative thoughts or feelings that are initiated or exacerbated by the trauma, and increased arousal and reactivity after exposure to the traumatic event. As many as $80 \%$ of EMS per- sonnel develop some symptoms of PTSD, even if they do not develop the full clinical syndrome. ${ }^{1,2,6}$ Symptoms such as anger, hostility, sleep disturbances, and avoidance can significantly interfere with individuals' personal and professional lives. ${ }^{2}$ The high-stress environment of EMS work also poses health risks such as obesity, sleep problems, and cardiovascular disease. ${ }^{7}$

Kerai and colleagues reported that among 518 EMS personnel, PTSD was associated with dysfunctional coping styles, anxiety, and depression. ${ }^{8}$ A sense of coherencethe perception of stressors and challenges as "manageable, meaningful, and comprehensible"9 - serves as a protective factor for EMS personnel in that individuals who report higher levels of a sense of coherence endorse fewer PTSD symptoms. ${ }^{9,10}$ Despite the literature on risk factors for PTSD among EMS personnel, ${ }^{1,2,8}$ a paucity of literature examines protective factors, other than a sense of coherence, that could be used to identify resilient EMS personnel. 
Strategies need to be developed to select resilient personnel and to foster resilience in existing EMS personnel.

The concept of grit is proposed to be a construct related to achievement and is worth study as a possible protective factor. In the definition by Duckworth et al, grit has 2 elements: persistence of effort in the face of adversity (perseverance) and consistency of interest (passion for longterm goals). ${ }^{11}$ Grit entails "working strenuously toward challenges, maintaining effort and interest over years despite failure, adversity, and plateaus in progress." 11 Duckworth and colleagues have found that grit is associated with retention in a variety of settings, including the military, sales representative jobs, graduating from high school, and remaining married. ${ }^{12}$ Although Duckworth et al do not discuss grit in terms of resilience in their articles, one hypothesis for these findings may be that individuals who have higher grit scores are more resilient, and this resilience enables them to overcome obstacles to reach their goals. Although relatively little work has empirically examined the relationship between grit and resilience, 2 studies have reported that the construct of grit is associated with lower rates of burnout and greater psychological well-being among medical residents. ${ }^{13,14}$ Further, in a survey of 548 physicians in the United Kingdom, a negative correlation between grit and burnout was reported. ${ }^{15}$ These studies lend support to the hypothesis that individuals with higher grit may also be more resilient.

The current study examined the relationship between grit and resilience among EMTs/paramedic workers. We hypothesized that those who rated themselves higher on the grit scale would report fewer PTSD symptoms, indicating greater resilience to the impact of work stressors. A secondary hypothesis was that grit is related to coping mechanisms, which are related to resilience. ${ }^{16,17}$

\section{METHODS}

The authors developed an online survey for this study. Because the purpose of the study was to examine the impact of their work on EMS personnel, we chose measures that would assess exposure to traumatic events, PTSD symptoms, and coping mechanisms. When possible, we chose psychometrically sound abbreviated versions of scales to minimize the time required to complete the survey. A link to the survey was sent to East Baton Rouge Parish Emergency Medical Services personnel via an email distribution list. The survey was available from June 2017 to August 2017. A reminder email was sent at the end of June. Participants were informed that the survey was voluntary and anonymous, including the inability to capture IP addresses, and that participation implied consent. This study was approved by Our Lady of the Lake College Institutional Review Board, now the Franciscan Missionaries of Our Lady University Institutional Review Board.

\section{Measures}

Demographic variables and the following self-report measures were assessed: the PTSD Checklist for DSM-5 (PCL-5), ${ }^{18}$ the Grit Scale, ${ }^{11}$ the Brief COPE scale, ${ }^{19}$ and the Professional Exposure to Traumatic Experiences scale (modified from the Life Events Checklist). ${ }^{20}$ Demographic variables assessed included age (categorized by decade of life), sex, and years on the job.
The PCL-5 contains 20 items rated on a 0 ("not at all") to 4 ("extremely") scale that assess how much the individual has been bothered by symptoms of PTSD based on the Diagnostic and Statistical Manual for Mental Disorders, Fifth Edition (DSM-5) within the past month. ${ }^{5}$ Higher scores indicate more self-reported PTSD symptoms. The PCL- 5 has demonstrated excellent internal consistency $($ alpha $=0.94)$ and good test-retest reliability $(r=0.82) .{ }^{21}$

The 12-item Grit Scale contains 6 items assessing consistency of interest and 6 items assessing perseverance of effort. Items related to consistency of interest include "My interests change from year to year" and "I often set a goal but later choose to pursue a different one." Items related to perseverance include "I finish whatever I begin" and "Setbacks don't discourage me." Items are rated on a 1 to 5 scale with answer choices ranging from "not at all like me" to "very much like me." Higher scores are indicative of higher self-reported grit. The 12-item Grit Scale has demonstrated high internal consistency (alpha $=0.85){ }^{11}$

The Brief COPE scale ${ }^{19}$ includes 28 items rated on a 0 to 3 scale ranging from "I do not do this" to "I do this a lot." Example items include "I turn to other activities to take my mind off things," "I get emotional support from others," and "I get help and advice from other people." The items are categorized into 14 scales with 2 items each: self-distraction, active coping, denial, substance abuse, use of emotional support, use of instrumental support, behavioral disengagement, venting, positive reframing, planning, humor, acceptance, religion, and self-blame. Higher scales scores indicate that individuals are more likely to use the coping mechanism. Scales of the Brief COPE have demonstrated adequate internal consistency, ranging from alpha $=0.50$ for venting to alpha $=0.90$ for substance abuse. ${ }^{19}$

The Professional Exposure to Traumatic Experiences scale assesses 11 items that first responders may be exposed to. Example items include "serious accident at work, home or during a recreational activity," "sudden violent death (eg, homicide or suicide)," and "fire or explosion." Individuals rate how often they are exposed to each of the 11 items on a 5-point scale ranging from "never" to "all the time." Higher scores are indicative of higher frequencies of self-reported exposure to traumatic events. The items were adapted from the Life Events Checklist. ${ }^{20}$

\section{Statistical Analyses}

Descriptive statistics were calculated. Pearson correlations measured relationships between demographic variables, professional exposure, Grit Scale scores, and PCL-5 scores. Linear regression analysis was conducted to determine whether demographic variables (ie, age, sex, and years of experience) or professional exposure predicted Grit Scale scores. A second linear regression analysis examined whether Grit Scale scores, professional exposure, and demographic variables predicted PCL-5 scores. Correlations between Brief COPE scales, PCL-5, and Grit Scale scores were examined using Pearson correlations. To determine whether Grit Scale scores added uniquely to the prediction of PCL-5 scores, we conducted a hierarchical linear regression with the 14 Brief COPE scale scores as step 1 and total Grit Scale score as step 2. A power analysis indicated that linear regression including 15 predictors (ie, the Grit Scale and 14 Brief COPE scales) in our sample of 84 
Table 1. Demographic Information for the Emergency Medical Service Personnel Who Responded to our Survey

\begin{tabular}{|c|c|}
\hline Variable & Frequency (\%) \\
\hline \multicolumn{2}{|l|}{ Sex } \\
\hline Male & $47(56)$ \\
\hline Female & $37(44)$ \\
\hline \multicolumn{2}{|l|}{ Race } \\
\hline Caucasian & $80(95.2)$ \\
\hline Other & $4(4.8)$ \\
\hline \multicolumn{2}{|l|}{ Age, years } \\
\hline $21-30$ & $14(16.7)$ \\
\hline $31-40$ & $29(34.5)$ \\
\hline $41-50$ & $27(32.1)$ \\
\hline $51-60$ & $12(14.3)$ \\
\hline $60+$ & $2(2.4)$ \\
\hline \multicolumn{2}{|l|}{ Training ${ }^{a}$} \\
\hline EMT basic & $6(7.2)$ \\
\hline EMT intermediate & $1(1.2)$ \\
\hline EMT paramedic & $76(91.6)$ \\
\hline \multicolumn{2}{|l|}{ Years on the job } \\
\hline $0-2$ & $1(1.2)$ \\
\hline $3-5$ & $12(14.3)$ \\
\hline $6-10$ & $13(15.5)$ \\
\hline $11-15$ & 15 (17.9) \\
\hline$>15$ & $43(51.2)$ \\
\hline
\end{tabular}

a One individual did not respond to the question regarding training. EMT, emergency medical technician.

individuals yielded a power of 0.91 to detect large effect sizes $\left(R^{2}=0.26\right)$. The significance level for all analyses was set at $P<0.05$.

\section{RESULTS}

The survey link was emailed to 170 EMS personnel. In all, 108 individuals completed the demographic questions. Ninety individuals completed the Brief COPE scales, except for the religion scale that 89 individuals completed. Eighty-four individuals completed the Grit Scale and the PCL-5. Therefore, statistical analyses were performed on the 84 individuals who completed both the Grit Scale and the PCL-5.

The mean PCL-5 symptom severity score was 22.55 $(S D=17.44)$, and the mean Grit Scale score was 3.46 $(S D=0.59)$. All 84 individuals responded to the questions regarding professional exposure to traumatic experiences, and the mean score was $25.20(S D=6.43)$. Demographic data are presented in Table 1.

\section{Correlation With Demographic Variables}

PCL-5 scores were significantly and negatively correlated with age $(r=-0.22, n=84, P<0.05)$ but not with years on the job $(r=-0.17, n=84, P=0.12)$, sex $(r=0.04, n=84$, $P=0.71$ ), or professional exposure to traumatic experiences $(r=0.10, n=84, P=0.36)$. Grit Scale scores were not
Table 2. Correlations between PCL-5 and Grit Scale Scores and BRIEF Cope Scale Scores

\begin{tabular}{lcc}
\hline \multicolumn{1}{c}{ Scale } & PCL-5 & Total Grit Scale Score \\
\hline Total Grit Scale score & $-0.568^{* * *}$ & 1.00 \\
BRIEF Cope scale scores & & \\
Self-distraction & $0.266^{* *}$ & $-0.252^{*}$ \\
Active coping & -0.206 & $0.354^{* * *}$ \\
Denial & $0.219^{*}$ & $-0.288^{*}$ \\
Substance abuse & $0.510^{* *}$ & $-0.361^{* *}$ \\
Emotional support & $-0.255^{*}$ & 0.125 \\
Instrumental support & -0.095 & -0.001 \\
Disengagement & $0.580^{* * *}$ & $-0.334^{* *}$ \\
Venting & $0.271^{* *}$ & -0.149 \\
Reframing & -0.125 & 0.163 \\
Planning & 0.079 & 0.124 \\
Humor & 0.088 & 0.057 \\
Acceptance & -0.131 & $0.266^{* *}$ \\
Religion & 0.198 & $-0.242^{*}$ \\
Self-blame & $0.521^{* * *}$ & $-0.248^{*}$ \\
\hline
\end{tabular}

PCL-5, Posttraumatic Stress Disorder Checklist for the Diagnostic and Statistical Manual of Mental Disorders-5.

Note: ${ }^{\star} P<0.05,{ }^{\star \star} P<0.01,{ }^{\star \star \star} P<0.001$.

significantly correlated with age $(r=-0.19, \mathrm{n}=84, P=0.08)$, years on the job $(r=-0.18, \mathrm{n}=84, P=0.11)$, sex $(r=-0.20$, $\mathrm{n}=84, P=0.07$ ), or professional exposure to traumatic experiences $(\mathrm{r}=-0.06, \mathrm{n}=84, P=0.58)$. Linear regression revealed that none of the demographic variables or professional exposure was statistically significantly predictive of the total Grit Scale score (adjusted $\mathrm{R}^{2}=0.21, \mathrm{~F}(4,79)=1.45, P<0.22$ ). A second linear regression that included the total Grit Scale score and demographic variables revealed that only the Grit Scale score significantly predicted PCL-5 scores (adjusted $\left.\mathrm{R}^{2}=0.35, \mathrm{~F}(5,78)=8.37, P<0.001\right)$.

\section{Predicting Posttraumatic Stress Disorder Symptoms}

Table 2 presents a correlation matrix including the Brief COPE scales, the total Grit Scale score, and the PCL-5 score. The PCL-5 score was significantly negatively correlated with the Grit Scale score and emotional support. The PCL-5 score was significantly positively correlated with self-distraction, denial, substance abuse, disengagement, venting, and self-blame. The Grit Scale score was significantly negatively correlated with self-distraction, denial, substance abuse, disengagement, religion, and self-blame. The Grit Scale score was significantly positively correlated with active coping and acceptance. Five of the Brief COPE scales were significantly and negatively correlated with the Grit Scale score and positively correlated with the PCL-5 score.

Hierarchical linear regression established that PCL-5 scores were statistically significantly predicted by Brief COPE scale scores (step 1: $F(14,68)=8.59, P<0.001$ ). Overall, the 14 Brief COPE scale scores accounted for $56.4 \%$ of the variance. In step 2, total Grit Scale score 
Table 3. Final Multiple Regression Model of the Effects of Coping and Grit Scale Scores on PCL-5 Scores

\begin{tabular}{lccc}
\hline \multicolumn{1}{c}{ Model } & $\boldsymbol{\beta}$ & $\boldsymbol{t}$ & $\boldsymbol{P}$ Value \\
\hline Disengagement & 0.393 & 4.266 & 0.000 \\
Self-blame & 0.34 & 3.352 & 0.001 \\
Total Grit Scale score & -0.295 & -3.127 & 0.002 \\
Substance abuse & 0.271 & 3.165 & 0.002 \\
Denial & -0.185 & -2.098 & 0.040 \\
Planning & -0.168 & -1.712 & 0.092 \\
Emotional support & -0.134 & -1.481 & 0.143 \\
Humor & 0.095 & 1.278 & 0.206 \\
Religion & -0.047 & -0.586 & 0.560 \\
Instrumental support & 0.046 & 0.406 & 0.686 \\
Venting & 0.042 & 0.538 & 0.593 \\
Acceptance & 0.039 & 0.407 & 0.685 \\
Active coping & 0.028 & 0.295 & 0.769 \\
Reframing & 0.006 & 0.072 & 0.943 \\
Self-distraction & -0.002 & -0.027 & 0.978 \\
\hline
\end{tabular}

PCL-5, Posttraumatic Stress Disorder Checklist for the Diagnostic and Statistical Manual of Mental Disorders-5.

was added to the equation, and the model was statistically significant (adjusted $R^{2}=0.617, F(15,67)=9.81$, $P<0.001)$. The total Grit Scale score contributed an additional $4.8 \%$ of the variance (adjusted $R^{2}$ change $=0.048$; $\left.\mathrm{F}_{\text {change }}(1,67)=10.35, P<0.01\right)$. Examination of the coefficients revealed that only the total Grit Scale score $(\beta=-0.295$, $\mathrm{t}=-3.127, P=0.002)$ and the following Brief COPE scale scores were significant predictors of PCL-5 score: denial $(\beta=-0.185, \mathrm{t}=-2.098, P=0.04)$, substance abuse $(\beta=0.271$, $\mathrm{t}=3.165, P=0.002)$, disengagement $(\beta=0.393, \mathrm{t}=4.266$, $P<0.001)$, and self-blame $(\beta=0.34, \mathrm{t}=3.352, \quad P=0.001)$ (Table 3).

\section{DISCUSSION}

Our study examined the relationship among grit, coping mechanisms, demographic variables, professional exposure, and self-reported PTSD symptoms among EMS workers, with an interest in understanding the contribution of grit to resilience as measured by PCL- 5 self-reported symptoms. Our study had 3 key findings. First, higher Grit Scale scores were significantly predictive of lower PCL-5 scores. Second, the Grit Scale score was significantly correlated with 8 scales of the Brief COPE. Of the 8 scales correlated with grit, 5 scales were also significantly correlated with PCL-5 scores. Interestingly, these 5 scales measured maladaptive coping mechanisms that were negatively correlated with Grit Scale score and positively correlated with PTSD symptoms. Finally, hierarchical linear regression analysis revealed that Grit Scale score was significantly and uniquely predictive of PTSD symptoms after controlling for coping mechanisms.

The mean Grit Scale score for EMS personnel in our sample was 3.46. This score is consistent with means for adults aged 25 years and older in the research by Duckworth et al. ${ }^{11}$ Therefore, as a whole, EMS personnel do not appear to demonstrate significantly more or less grit than the general population.

Our results indicate a significant relationship between higher Grit Scale scores and lower self-reported PTSD symptoms in EMS personnel. There are several possible explanations for why individuals who indicate higher grit tend to be more resilient. Individuals who report more grit may develop mechanisms for coping with adversity that foster well-being and protect against psychopathology. Alternatively, resilient individuals are those who persist in the face of obstacles and continue to pursue their passions, so their resilience may lead to greater grit over time. Further studies are necessary to understand the impact of the construct of grit on resilience. Despite the preliminary nature of our findings, they are consistent with data indicating that Grit Scale scores of medical residents predict lower burnout and greater psychological well-being 6 months after the baseline assessment ${ }^{13}$ and that higher Grit Scale scores are associated with lower surgical resident attrition. ${ }^{14}$

An additional implication of the current data is that EMTs/paramedic workers with lower Grit Scale scores may need additional support, including skills training to enhance coping strategies. This study demonstrated clear relationships between disengagement, self-blame, substance abuse, self-distraction, and denial as coping mechanisms with increased reported PTSD symptom severity and decreased grit. Alternatively, emotional support was negatively associated with PTSD symptoms. Incorporating informal debriefing sessions into postcall routines may be an important mechanism for bolstering resilience among EMS personnel.

Limitations of the current study include a response rate of $49 \%$. Because we only used self-report questionnaires, we were unable to determine whether individuals met clinical criteria for PTSD. Further, EMTs/paramedic personnel were selected from only one employer that agreed to cooperate with the research team, limiting the generalizability of these findings.

\section{CONCLUSION}

To our knowledge, our results are the first to examine the relationship between grit and PTSD symptoms. Our findings suggest that EMTs/paramedics with greater grit tend to endorse fewer symptoms of PTSD. The implication of this finding is that grit is associated with resilience. Further, our preliminary data suggest that grit is related to, but distinct from, other constructs that predict resilience, such as coping mechanisms. Large prospective studies could have significant implications for EMT/paramedic hiring practices and for strategies to build grit in existing EMT/paramedic personnel to bolster resilience.

\section{ACKNOWLEDGMENTS}

The authors have no financial or proprietary interest in the subject matter of this article.

An abstract entitled "The Relationship between Grit and Resilience in Emergency Medical Service Personnel" was presented at the LSU Health Regional Research Day in Baton Rouge, LA in May 2018. 


\section{REFERENCES}

1. Alexander DA, Klein S. Ambulance personnel and critical incidents: impact of accident and emergency work on mental health and emotional well-being. Br J Psychiatry. 2001 Jan;178(1):76-81.

2. Lewis-Schroeder NF, Kieran K, Murphy BL, Wolff JD, Robinson MA, Kaufman ML. Conceptualization, assessment, and treatment of traumatic stress in first responders: a review of critical issues. Harv Rev Psychiatry. 2018 Jul/Aug;26(4):216-227. doi: 10.1097/HRP.0000000000000176.

3. Petrie K, Milligan-Saville J, Gayed A, et al. Prevalence of PTSD and common mental disorders amongst ambulance personnel: a systematic review and meta-analysis. Soc Psychiatry Psychiatr Epidemiol. 2018 Sep;53(9):897-909. doi: 10.1007/s00127-018-1539-5.

4. Berger W, Coutinho ES, Figueira I, et al. Rescuers at risk: a systematic review and meta-regression analysis of the worldwide current prevalence and correlates of PTSD in rescue workers. Soc Psychiatry Psychiatr Epidemiol. 2012 Jun;47(6):1001-1011. doi: 10.1007/s00127-011-0408-2.

5. American Psychiatric Association. Diagnostic and Statistical Manual of Mental Disorders (DSM-5). Washington, DC: American Psychiatric Association Publishing; 2013.

6. Mishra S, Goebert D, Char E, Dukes P, Ahmed I. Trauma exposure and symptoms of post-traumatic stress disorder in emergency medical services personnel in Hawaii. Emerg Med J. 2010 Sep;27(9):708-711. doi: 10.1136/emj.2009.080622.

7. Hegg-Deloye S, Brassard P, Jauvin N, et al. Current state of knowledge of post-traumatic stress, sleeping problems, obesity and cardiovascular disease in paramedics. Emerg Med J. 2014 Mar;31(3):242-247. doi: 10.1136/emermed-2012-201672.

8. Kerai SM, Khan UR, Islam M, Asad N, Razzak J, Pasha O. Post-traumatic stress disorder and its predictors in emergency medical service personnel: a cross-sectional study from Karachi, Pakistan. BMC Emerg Med. 2017 Aug 29;17(1):26. doi: 10.1186/s12873-017-0140-7.

9. Jonsson A, Segesten K, Mattsson B. Post-traumatic stress among Swedish ambulance personnel. Emerg Med J. 2003;20(1):79-84.
10. Streb M, Häller P, Michael T. PTSD in paramedics: resilience and sense of coherence. Behav Cogn Psychother. 2014 Jul;42(4):452-463. doi: 10.1017/S1352465813000337.

11. Duckworth AL, Peterson C, Matthews MD, Kelly DR. Grit: perseverance and passion for long-term goals. J Pers Soc Psychol. 2007 Jun;92(6):1087-1101.

12. Eskreis-Winkler L, Shulman EP, Beal SA, Duckworth AL. The grit effect: predicting retention in the military, the workplace, school and marriage. Front Psychol. 2014 Feb 3;5:36. doi: 10.3389/fpsyg.2014.00036.

13. Salles $A$, Cohen GL, Mueller CM. The relationship between grit and resident well-being. Am J Surg. 2014 Feb;207(2):251-254. doi: 10.1016/j.amjsurg.2013.09.006.

14. Salles $A$, Lin D, Liebert $C$, et al. Grit as a predictor of risk of attrition in surgical residency. Am J Surg. 2017 Feb;213(2):288-291. doi: 10.1016/j.amjsurg.2016.10.012.

15. Halliday L, Walker A, Vig S, Hines J, Brecknell J. Grit and burnout in UK doctors: a cross-sectional study across specialties and stages of training. Postgrad Med J. 2017 Jul;93(1101):389-394. doi: 10.1136/postgradmedj-2015-133919.

16. Southwick SM, Vythilingam M, Charney DS. The psychobiology of depression and resilience to stress: implications for prevention and treatment. Annu Rev Clin Psychol. 2005;1:255-291. doi: 10.1146/annurev.clinpsy.1.102803.143948.

17. Zeidner M, Endler NS. Handbook of Coping: Theory, Research, Applications. Hoboken, NJ: John Wiley \& Sons, Inc; 1996.

18. The PTSD Checklist for DSM-5 (PCL-5). U.S. Department of Veterans Affairs. www.ptsd.va.gov/professional/assessment/ adult-sr/ptsd-checklist.asp. Accessed November 12, 2018.

19. Carver CS. You want to measure coping but your protocol's too long: consider the brief COPE. Int J Behav Med. 1997;4(1):92-100. doi: 10.1207/s15327558ijbm0401_6.

20. Gray MJ, Litz BT, Hsu JL, Lombardo TW. Psychometric properties of the life events checklist. Assessment. 2004 Dec;11(4):330-341. doi: 10.1177/1073191104269954.

21. Blevins CA, Weathers FW, Davis MT, Witte TK, Domino JL. The posttraumatic stress disorder checklist for DSM-5 (PCL-5): development and initial psychometric evaluation. J Trauma Stress. 2015 Dec;28(6):489-498. doi: 10.1002/jts.22059.

This article meets the Accreditation Council for Graduate Medical Education and the American Board of Medical Specialties Maintenance of Certification competencies for Medical Knowledge and Interpersonal and Communication Skills. 